\title{
Our Readers Write ...
}

'General Relativity and the Accelerated Expansion of the Universe' by Patrick Das Gupta, in the March 2012 issue of Resonance is an excellent article. He has analyzed significantly the core of the subject though they are extremely difficult to understand and has made them easy to comprehend; the style of his writing and its phrase is like reading a detective story and the finishing touch "The riddle of cosmological constant persists even though the original motivation for its introduction has long vanished after the discovery of Hubble's law. It is like the smile of the Cheshire cat- the cat disappeared but its smile remains" is a punch line.

Something I want to emphasize: Though we are living in a quantum world which occupies every part of our lives the world of physicists' community still depends only on GR which is based on classical mechanics to analyze the equivalence principles. Till his last breath, Albert Einstein did not believe that quantum mechanics is fundamental as it is based on uncertainty principles; but Einstein believed strongly in determinism; so that he said 'God does not play dice'. Here God means the Laws of Nature.

The longing for Quantum Gravity may be a mirage we follow, and the only hope of physicists is the String Theory which describes everything well but not testable due to its 10 dimensions, single dimension of string and its Planck scale of $10^{-33} \mathrm{~cm}$ for which one needs an accelerator, the size of our solar system, that is not practicably viable. In 1929 the static universe became expanding universe; and in 1998 the expanding universe became accelerating universe and who knows what will happen to the accelerating universe eventually?

N Sithu Muruganandam

Founder - Einstein Science Movement Email: sithu.muruganandam@gmail.com

I was happy to read the April 2012 issue of Resonance, especially the editorial. I liked both points of content textbooks and school level. It is close to my heart since I have been involved in school textbook writing and teaching.

I think the main content of Resonance should be the education aspect of science. Varied content can be included, such as research papers and articles based on variety of small and big experiments of teaching-learning of science in the classroom and outside, impact of administrative efforts for enhancing student participation, studies of textbook contents, and curricula, preparation of learning materials, ICT use, institution-wide efforts of understanding and appreciating science and so on.

With due respect to all teachers, I feel that articles on teaching methodology can be useful especially for teachers of higher education, who never get to learn pedagogy.

The policy level efforts of encouraging science education and research would go in vain if not supported with ample and spirited interactions among various stakeholders of education. The interactions need to be vertical and horizontal and Resonance can play an important role in it.

Lalita Agashe

Email:lalitaagashe@gmail.com 